\title{
ÉTICA Y POLÍTICA EN LA INVESTIGACIÓN DEL TURISMO
}

ALEJANDRO MANTECÓN alejandro.mantecon@ua.es Universidad de Alicante, España

El cuestionamiento de las lógicas positivistas, dominantes en la investigación del turismo, favoreció en el umbral del siglo XXI el desarrollo del llamado giro crítico de los estudios turísticos y el intento de construcción de un nuevo paradigma con rasgos distintivos: la Academia de Esperanza. Se revisa este proceso y se critican las bases teóricas y los supuestos ético-políticos en los que se sostiene. Finalmente, se discute el tipo de relación que debe establecer el análisis crítico del turismo con la reflexión moral sobre sus efectos.

Palabras clave: reflexividad, empirismo abstracto, acumulación de conocimiento, giro crítico, epistemología.

\section{ETHICS AND POLITICS IN TOURISM RESEARCH}

The questioning of positivist logics, dominant in tourism research, drove to the so-called critical turn in tourism studies, on the threshold of the twenty-first century. As a result, the Academy of Hope is an attempt to build a new paradigm with distinctive features. This process is reviewed, with a critic of the theoretical basis and ethic-political foundations. Finally, the relationship that must be established between the critical tourism analysis and the moral reflection on its effects is discussed.

Keywords: reflexivity, abstract empiricism, knowledge accumulation, critical turn, epistemology. 


\section{Introducción}

a investigación y la reflexión acerca de las repercusiones de la actividad turística fueron empresas intelectuales poco frecuentes, hasta que, una vez concluida la Segunda Guerra Mundial, la expansión de las sociedades de consumo en la Europa capitalista y Norteamérica propició la eclosión del turismo de masas.

En los años 50 y 60 se produjo un tímido impulso, gracias a la publicación, todavía muy ocasional, de libros introductorios o de revistas como Tourism: an International Interdisciplinary Journal (fundada en 1953), Estudios Turísticos (de 1964) o Journal of Travel Research (de 1968). No obstante, es difícil encontrar artículos de esa época que presenten el turismo como un fenómeno conflictivo.

En la década de los 70, un grupo de pioneros bosqueja otros enfoques, ajenos a los discursos apologéticos emitidos por los organismos oficiales. Estos se concretan en ensayos redactados desde perspectivas antropológicas y sociológicas críticas (De Kadt, 1979; Estivill, 1979; Gaviria, 1974; Galán et al., 1977; Greenwood, 1977; Jurdao, 1979; Nash, 1977; Turner y Ash, 1975), algunas influenciadas por distintas corrientes neomarxistas (teorías de la dependencia o pensamiento urbano de Henri Lefebvre).

La profusión de publicaciones de los años 80 y 90 del siglo pasado trastoca la situación anterior, con dos efectos evidentes: a) la legitimación del turismo como un objeto de estudio complejo y relevante para las ciencias sociales, y b) su creciente incorporación a las universidades a través de la implementación de programas formativos, la creación de grupos de trabajo y un incremento de las líneas de financiación. Por supuesto, el proceso se extiende de diversas maneras y con ritmos muy dispares, reconociéndose hoy dinámicas consolidadas junto a otras apenas incipientes.

La institucionalización académica de la investigación turística queda asociada a debates epistemológicos y, sobre todo, a disputas corporativistas que reivindican la apropiación del turismo por parte de las áreas de conocimiento, departamentos o facultades con más arraigo e influencia. Así, entre las aspiraciones de unos y las demostraciones de poder de otros, se originan discusiones recurrentes a propósito de si el turismo posee una entidad disciplinaria genuina o si, más bien, es un espacio distinto que se abre a la indagación geográfica, antropológica, mercadotécnica, entre otras (Leiper, 2000; Tribe, 1997; 2000). 
Al volver la vista atrás y observar los caminos transitados durante las dos primeras décadas del nuevo milenio, es posible advertir cómo el examen del turismo se adentra en una nueva etapa con rasgos distintivos. En su intento por trascender los problemas de identidad de los estudios turísticos, las aproximaciones más vanguardistas proponen esquemas interpretativos posdisciplinares (Coles et al., 2006), capaces de configurar redes (de investigadores, ideas, conceptos) conectadas entre sí con el fin de desarrollar proyectos que encaren el turismo no como un sistema más o menos determinante, sino como una dimensión constitutiva de las sociedades modernas (Darbellay y Stock, 2012).

La realidad turística se convierte entonces en un campo de trabajo central y paradigmático en el que, junto a las miradas más convencionales, confluyen y se interpenetran enfoques como los estudios de género (Aitchison, 2005; Cole, 2018; Figueroa-Domecq et al., 2015), la ecología (Becken y Hay, 2007; Scott, 2008), la investigación de las emociones (Picard y Robinson, 2012), la teoría del actor-red y el estudio de los vínculos que los turistas establecen con los objetos materiales (Beard et al., 2016; Muecke y Wergin, 2014), el paradigma de la movilidad (Sheller y Urry, 2006; Urry, 2007), los análisis sobre la digitalización de la vida social (Huete, 2019; Wang et al., 2002, 2012), o la filosofía y la ética del turismo (Fennell, 2006; Tribe, 2009).

Estas perspectivas tienden a empapar los modos en los que se plantea el entendimiento tanto de las prácticas turísticas tradicionales como de la infinidad de formas emergentes; además, atraviesan los grandes temas que han focalizado la curiosidad de los expertos: los tipos de turistas (motivaciones, comportamientos), los impactos de la actividad turística (económicos, socioculturales, geoambientales), las interacciones entre los visitantes y los residentes en las sociedades receptoras (y las percepciones e imaginarios que de ellas resultan), o la evolución de la industria que promueve el negocio del ocio y el viaje (cambios en las estructuras empresariales y laborales).

Sin duda, la comprensión del hecho turístico se ha incrementado y refinado. A la vez, surgen nuevos retos como consecuencia de las transformaciones experimentadas por el contexto tecnológico, las crisis recurrentes del capitalismo, los riesgos inherentes al calentamiento global o, en el momento de escribir estas líneas, el desastre provocado por la pandemia del COVID-19. Otro tipo de dificultades aluden a asuntos que se vienen arrastrando por décadas y que, más que resolverse, se adap- 
tan a los nuevos tiempos. También se hace referencia a tensiones ligadas al envoltorio político-ideológico que interviene en la producción de conocimiento sobre el turismo.

Una de esas tensiones es analizada por Claudio Milano (2017), quien aborda los motivos que explican el carácter subalterno de la literatura redactada en español y portugués acerca de la antropología del turismo, frente a aquella escrita en inglés. Los argumentos expuestos por Milano son extrapolables a otras disciplinas, y se enmarcan en la panorámica de desafíos examinados por Maribel Osorio y Maximiliano Korstanje (2017), muchos de los cuales están emparentados con los objetivos de este texto, pues también explora la construcción social del conocimiento.

\section{Objetivo}

En las próximas páginas, se invita a pensar acerca del modo en el que los estudios turísticos se ven afectados debido a una tensión consustancial al trabajo científico en general y al científico-social en particular: la influencia de la reflexividad en la investigación. Por reflexividad se entiende la cualidad de las personas de dotar de diferentes significados a las acciones que emprenden, así como las circunstancias que las influyen y, en consecuencia, modificar sus actitudes y conductas.

Esta vieja cuestión es gestionada de distintas maneras en unos y otros entornos. Para el caso del turismo, ha sido revisada desde distintos ángulos; por ejemplo, son destacables las contribuciones de Tobar-Bustamante et al. (2015) o las de Castillo-Nechar y Panosso (2010). Aquí se atrae la atención a algunos de los problemas derivados de:

a) La pretensión de dotar a los estudios turísticos de un estatus científico, sostenido en supuestos positivistas simplificadores, que obvian las implicaciones de la reflexividad en el análisis científico-social.

b) La manera en que el denominado giro crítico (critical turn) de los estudios turísticos ha intentado superar el fetichismo metodológico y el reduccionismo analítico inherentes a una aplicación radical de los supuestos positivistas en la investigación del turismo. 


\section{La aspiración científica}

La ciencia es una estrategia de producción de conocimiento creada por los seres humanos en su deseo por entender el funcionamiento de ciertos aspectos de la realidad. Asume que existe una realidad cognoscible, que posee pautas de orden y regularidad descifrables, cuyo esclarecimiento nos proporciona la sensación de no vivir en un caos aleatorio y carente de sentido. A pesar de ser un proyecto social $y$, por lo tanto, de hallarse expuesto a múltiples contingencias, muestra una sorprendente eficacia a la hora de generar explicaciones sólidas de la realidad a la que pertenecemos.

Los hallazgos científicos parecen disociarse de los valores y las interpretaciones subjetivas, hasta convertirse en referentes de gran utilidad para organizar la vida. Por ejemplo, al unir dos átomos de hidrógeno con uno de oxígeno se forma una molécula de agua; en ausencia de elementos perturbadores la repetición de esta operación, guste o no, ofrecerá el mismo resultado.

Según la ortodoxia, el secreto de tal eficacia explicativa reside en una observación disciplinada, guiada por la aplicación del método científico. No importaría que la ciencia fuese una construcción colectiva, pues un individuo aislado, aplicando con rigurosidad el método científico, obtendría los mismos resultados que otro en condiciones idénticas de replicabilidad experimental.

Los problemas de esta concepción fueron desmenuzados por Thomas S. Kuhn (1962; 1977), y están relacionados con el hecho de que las decisiones que los científicos toman están mediadas por las estructuras grupales en las que se hallan insertos, a propósito de la definición de su objeto de estudio (¿qué es y qué no es un problema de investigación?) y de la posición que frente a él adoptan (¿qué parte de la realidad es accesible en términos científicos? y ¿cómo materializar ese acceso?). Es decir, la validez y fiabilidad del discurso científico no se sustenta en la aplicación aséptica de un método unívoco, sino en un conjunto de operaciones articuladas por compromisos cognoscitivos socialmente condicionados.

Las disputas relativas a estos planteamientos han hecho correr ríos de tinta (Popper, Lakatos, Feyerabend, Latour y un largo etcétera). En las ciencias sociales los problemas se multiplican, porque la naturaleza de las relaciones que se establecen entre el sujeto y el objeto de la investigación, así como los contextos que determinan esas 
relaciones, amplifican de tal modo las inquietudes que, desde algunos ámbitos, se cuestiona que las ciencias sociales merezcan el calificativo de ciencias.

A continuación, se exponen algunas ideas sobre la relación sujeto-objeto (y sus repercusiones en los estudios turísticos), enlazadas posteriormente con los factores contextuales.

Florian Znaniecki (1927) Ilamó coeficiente humanístico a la propiedad de los sistemas culturales en virtud de la cual el conocimiento de la realidad social solo podría alcanzarse si el investigador admite que su objeto de estudio no posee una independencia (una resistencia al cambio) comparable a la de los objetos a los que se enfrentan los científicos naturales. En el análisis de una situación social, el experto tendría que abordar las claves interpretativas (subjetivas y volubles) que construyen los diferentes actores en ella involucrados, y distinguir esas interpretaciones de la suya.

Ahondando en esta cuestión, Henry A. Landsberger (1958) acuña la expresión efecto Hawthorne, al revisar los estudios dirigidos por Elton Mayo en la planta industrial Western Electric's Hawthorne Works de Chicago, realizados entre 1924 y 1932. El efecto Hawthorne se refiere a la distorsión que se produce en la investigación debido al conocimiento que tienen los sujetos estudiados del hecho de serlo (y la consiguiente variación en sus actitudes y comportamientos).

Estos factores, la subjetividad y la autoconciencia, son la base en la que se asienta la reflexividad característica de las interacciones humanas. Aceptar su existencia hace casi imposible edificar unas ciencias sociales equiparables a las ciencias naturales, pues las expectativas de acumulación de conocimiento (de acuerdo con la lógica de lo que Kuhn llamó ciencia normal) se ven muy restringidas. Pero esa acumulación es nada menos que la manifestación del progreso de la actividad científica en un campo dado (Kuhn, 1962).

Sin embargo, ni las ciencias sociales en general, ni los estudios turísticos en particular, encajan de buen grado las implicaciones de este dictamen. La matematización a la que se expusieron las ciencias sociales durante la centuria pasada constituye un monumental esfuerzo de afirmación de su estatus científico que, finalmente, cristaliza en una proliferación de estudios de caso sustentados en análisis estadísticos más o menos sofisticados. 
Estos estudios, en sus versiones más afortunadas, ofrecen teorías de alcance medio: modelos teóricos con una validez temporal determinada, útiles para explicar realidades en contextos concretos (Merton, 1949). En sus versiones más exasperantes, desembocan en un torrente de lo que Wright Mills (1959) denominó empirismo abstracto. En la investigación del turismo, esta dinámica acumulativa se puede apreciar con nitidez gracias a los estudios dedicados a analizar la valoración del turismo por parte de la sociedad receptora a la luz de la Teoría del Intercambio Social.

La idea central de este enfoque sugiere que los residentes apoyarán el desarrollo turístico, siempre y cuando perciban que los beneficios esperados serán mayores que los costos. Esta línea no ha producido generalizaciones empíricas relevantes, aparte de constatar que en los estudios de opinión sobre los efectos del turismo se identifica una mayor concentración de valoraciones positivas alrededor de las repercusiones económicas, a la par de una apreciación más crítica de los impactos medioambientales y socioculturales (Mantecón, 2016).

Además de amasar certezas estadísticas de dudosa relevancia y amontonar estudios de caso sin apenas conexión, en las inercias observadas se advierte una suerte de isomorfismo teórico, ajeno a cualquier cuestionamiento crítico de la literatura existente (aunque, desde luego, han alcanzado un éxito notable facilitando la vida a quienes buscan un catalizador de publicaciones).

El exceso de confianza que la Teoría del Intercambio Social deposita en la racionalidad individual, su infravaloración de los aspectos emocionales, la escasa atención que presta al cambio social, su dificultad para articular los análisis cuantitativos con diseños cualitativos, así como el desinterés por explicar la influencia que las relaciones de poder ejercen en la formación de la opinión pública, invitan a explorar vías alternativas que ayuden a entender las distintas maneras en que las personas se enfrentan al turismo (Mantecón y Velasco, 2020).

Las corporaciones empresariales y las administraciones públicas (por separado o en convergencia, al margen de disquisiciones epistemológicas) afinan su habilidad para combinar el empirismo abstracto con la practicidad liberal, reconvirtiendo a investigadores aparentemente independientes en una suerte de consultores (o de productores de una supuesta legitimidad científico-académica) dedicados no ya a elaborar estudios de mercado, sino a ensamblar microinformes con fines publicitarios o para facilitar corrientes de opinión. 
Dicho estado de las cosas parece abocar a una reproducción acrítica de las realidades ya conocidas y a una contención de las propuestas innovadoras. El desarrollo científico-social resultante de la lógica acumulativa causa decepción, y su proyección como impulsor de progreso no acaba de reconocerse. En la investigación del turismo, esta percepción provoca y justifica el llamado giro crítico en los estudios turísticos. En el siguiente apartado se discute este asunto.

\section{La promesa de un mundo mejor}

Hace un tiempo, quien firma estas líneas asistió a un seminario universitario en el que un acreditado conferenciante, investigador del turismo, afirmaba estar cansado de la ciencia convencional. A continuación, compartía con los oyentes la decisión de aprovechar su posición, a fin de implicarse en los siguientes años solo en proyectos que sirvieran para hacer un mundo mejor. El hartazgo de este académico había derivado en inquietudes existenciales: ¿y si la ciencia y sus estructuras sociales no sirven para desplegar el modelo ético de vida en el que le gustaría verse reflejado?, ¿no sería más conveniente invertir el orden de las prioridades?, ¿acaso no sería más pragmático pensar el orden moral deseable y, después, poner la ciencia al servicio de su consecución?

En el umbral del siglo XXI, el giro crítico de los estudios turísticos surge en un contexto intelectual y político orientado por la esperanza de ensanchar las fronteras de la justicia social y de frenar la influencia del neoliberalismo. Este movimiento se concreta en publicaciones en las que se razona la pertinencia de liberarse de una ciencia subordinada a las prácticas consumistas y organizada por burocracias universitarias que reproducen valores masculinos. Dicha contestación se materializaría en el turismo en la promoción de formas de conocimiento y prácticas participativas, transformadoras, emancipadoras, comprometidas, trascendentales, igualitarias, sostenibles, etcétera (ver, por ejemplo, Ateljevic et al., 2007a; Pritchard et al., 2011; Wilson et al., 2012).

Un texto ilustrativo de esta corriente es el de John Tribe (2007), que entronca el giro crítico de los estudios turísticos con la teoría crítica de la Escuela de Frankfurt, aludiendo a Max Horkheimer, Theodor Adorno, Herbert Marcuse y Jürgen Habermas, así como al interés de estos por profundizar en los aspectos ético-morales que intervienen en la actividad investigadora. Lo que no precisa es que la forma en la que este 
asunto es abordado por los autores a los que hace referencia difiere bastante y tiene efectos prácticos casi antagónicos. Los denominadores comunes mencionados por Tribe: la preocupación por las relaciones de poder, el papel de las ideologías y la manipulación cultural o la conciencia emancipadora, son gestionados de forma desigual.

En líneas generales, aquí se concuerda con la distinción que Axel Honneth (1987) propone entre el círculo interior de la teoría crítica (integrado por Horkheimer, Adorno y, de un modo más ambiguo, por Marcuse) y el círculo exterior (Franz Neumann, Otto Kirchheimer, Walter Benjamin y Erich Fromm). Este último, con planteamientos mucho más optimistas que el primero, desemboca en la obra de Habermas (1981), donde se muestra una firme confianza en las alternativas que pueden resultar de la liberación del potencial racionalizador que la acción comunicativa guarda en su seno, siempre y cuando las personas sean capaces de crear espacios de interacción que permitan su desarrollo (no en vano es reconocida una deuda con su trabajo por Pritchard et al., 2011).

Por el contrario, las ideas nucleares del primer círculo, contenidas en sus libros más relevantes (Horkheimer, 1947; Horkheimer y Adorno, 1947), no concluyen en ninguna praxis colectiva destinada a transformar la realidad, más allá de la resistencia que cada individuo pueda ofrecer a los cantos de sirena coreados por la alienante industria cultural (el último gran sistema productor de conformismo creado por el capitalismo).

En clara oposición a las tesis posteriores de Habermas, para Horkheimer y Adorno (1947) la racionalidad humana solo habría probado una convincente eficacia histórica a la hora de expresar su dominio sobre la naturaleza y, al fin y al cabo, sobre la propia humanidad. Ante este diagnóstico, cualquier propuesta emancipadora pasaría por la redefinición (reconciliación) de las relaciones que los seres humanos entablan con la naturaleza.

En la introducción al libro que contiene el texto de Tribe, sus editoras (Ateljevic et al., 2007b) manifiestan la voluntad de promover una Academia de Esperanza desde la cual desarrollar estudios críticos del turismo superadores de las limitaciones asociadas a la tradición de la Escuela de Frankfurt, a su juicio deudora en exceso de las teorías marxistas y, por lo tanto, poco apta para dar el salto desde el way of knowing al way of being; esto es, del análisis de la realidad a la adquisición de un compromiso en el que la investigación se supedite a la subversión de las estructuras opresoras que coartan las dinámicas igualitarias y la justicia social. 
Al tener en cuenta que el conjunto de la obra busca asentar los principios teóricos del giro crítico, estas argumentaciones merecen algunos comentarios: por un lado, se invoca la herencia del pensamiento frankfurtiano porque hace hincapié en el análisis crítico de los factores ideológico-culturales, frente al mayor determinismo económico-materialista del marxismo clásico; pero, por otro, se pretende afirmar una autonomía ante esa misma herencia, ya que se cuestiona su potencial transformador a causa de su encadenamiento al referido marxismo. Efectivamente, la Escuela de Frankfurt presta una mayor atención a la influencia de la superestructura cultural; sin embargo, como se explicaba, sus propuestas prácticas distan de ser homogéneas.

En cuanto a las dudas acerca de la voluntad transformadora de los enunciados marxistas originales, puede recordarse la famosa sentencia en la que Marx declara que el tiempo para interpretar el mundo debe dejar paso al momento de su transformación (Marx, 1888). Asimismo, en relación con el alcance del cambio propuesto, quizá a la Academia de Esperanza le parezca poco ambicioso un plan en el que toda la actividad intelectual se pone al servicio de la abolición de la propiedad privada, el derrocamiento del régimen burgués y la conquista del poder por el proletariado (Marx y Engels, 1848).

Ateljevic et al. (2007b) y Pritchard et al. (2011) expresan su interés por desligarse de etiquetas frankfurtianas o marxistas y, en su lugar, las reemplazan por otras de cosecha propia (critical turn, hopeful tourism enquiry, co-transformative learning, academy of hope o spiritual activism).

Aunque Pritchard et al. (2011) hacen una mención muy puntual a algunos de los estudios realizados en la década de los 70 desde enfoques críticos (citados en el tercer párrafo de este ensayo), prefieren desmarcarse de ellos y afirmarse como impulsoras de un cambio de paradigma que enfatiza el interés por los elementos simbólico-discursivos, propuestas contestatarias inequívocas y una terminología apropiada para causar impacto en los restringidos círculos académicos donde hoy se decide qué debe y qué no tenerse en cuenta.

Tribe (2007) espera hasta los años 90 para localizar los escasos antecedentes que le son de utilidad a fin de mostrar la herencia de la teoría crítica en los estudios turísticos. Una de esas publicaciones es la de Keith Hollinshead (1999), donde los conflictos vinculados a la actividad turística quedan diluidos en un estilo narrativo más cercano a la crítica literaria que al análisis crítico del turismo. En todo caso, el argumento que Hollinshead propone tiene más interés si se enmarca en una reflexión 
sobre los márgenes de libertad en los que se mueve el turista moderno (o posmoderno), que en la comprensión de las repercusiones del turismo en los destinos.

Es verdad que en otros trabajos referidos por Tribe se exponen críticas a los enfoques etnocentristas occidentales (Hollinshead, 1992); no obstante, en su afán por ensalzar los factores de la superestructura ideológica, queda desplazado a un segundo plano el análisis crítico de la influencia que el turismo ejerce en, por ejemplo, la destrucción de ecosistemas, la expansión de dinámicas urbanizadoras carentes de una adecuada planificación, el (mal) desarrollo de las economías agrarias locales, la precarización de las condiciones laborales de quienes trabajan en el sector (y no solo en las regiones del llamado Sur Global), o en la configuración de economías ilícitas que, en los casos más lamentables, fomentan la explotación sexual.

Asimismo, en su interés por distinguirse de los esquemas clásicos del marxismo, de afirmar unas señas de identidad libres de etiquetas pasadas, de adaptarse a la estética discursiva de los tiempos presentes y de situar la transformación de la sociedad en la cúspide de su proyecto (exorcizando cualquier sustrato metodológico de raíces positivistas), el giro crítico de los estudios turísticos sustituye unos determinismos por otros, sin lograr articular entre medias una crítica del turismo tan transgresora y renovadora como pretende.

Cualquier esfuerzo encaminado a formular una crítica tan radical como para autodenominarse progresista, debe cuestionar las bases de sus principios y, para el caso que nos ocupa, esto tiene que ver con la consideración de la viabilidad del propio hecho turístico.

Como señalaban Horkheimer y Adorno (1947), solo el pensamiento que ejerce violencia sobre sí mismo es lo suficientemente duro como para quebrar los mitos. En un mundo en el que la estabilidad social se persigue a través del cambio incesante y la acumulación convulsiva (Rosa, 2010), la emancipación a la que se refiere con tanta insistencia la Academia de Esperanza se logra transformando la agresiva relación que establecemos con la naturaleza.

La interiorización de la falsa dicotomía naturaleza-cultura hace que olvidemos que las personas también somos naturaleza, y que si no la salvamos a ella tampoco nos salvamos nosotros. En el discurso del llamado por Honneth círculo interior de la Escuela de Frankfurt, valorado por el propio Honneth, y por tantos otros, como un pensamiento pesimista y carente de esperanza, se abordan los problemas cruciales 
y se encuentran las soluciones implícitas para encarar el mayor desafío del siglo XXI: garantizar un planeta habitable para los seres humanos del siglo XXII.

Al respecto, es inaceptable que en la economía turística mundial, articulada por una industria de los transportes tan contaminante y dependiente de la extracción de recursos no renovables, pueda justificarse la sostenibilidad de una actividad que en 2019 registró mil quinientos millones de desplazamientos internacionales.

El uso interesado que se ha hecho de la expresión desarrollo sostenible ha favorecido que con los años se aproxime más a un concepto político y mercadotécnico que a uno científico (Igoe et al., 2010). Entre las formas de turismo tradicional y la pluralidad de tipologías emergentes, existe un denominador común: la contaminación y la perturbación de ecosistemas a causa de un gigantesco flujo de movimientos motivados por la búsqueda de experiencias de ocio.

La crítica del orden social existente va unida a la implantación de medidas en virtud de las cuales el turismo tendría que gestionarse según principios de decrecimiento, o ser sustituido por otro tipo de actividades económicas que no generen impactos tan agresivos. Pero el esfuerzo que requeriría la aplicación de esas medidas es percibido como absurdo o contraproducente por, quizá, demasiados actores del sistema turístico, y las pautas de consumo objeto de críticas están interiorizadas como no problemáticas por, quizá también, demasiados millones de individuos. Los límites de tolerancia que las personas están dispuestas a asumir poseen una flexibilidad portentosa.

Ante esta situación, diferentes organismos reguladores se dedican a poner parches en un proceso de degradación en el que propuestas como la de la Academia de Esperanza y su giro crítico se autoproclaman en alternativas salvíficas, cuya principal característica es la insustancialidad y el solipsismo académico.

El análisis de los discursos, muy necesario para entender las razones profundas que orientan los puntos de vista de unos y otros actores, se diluye en reflexiones en torno al envoltorio simbólico del turismo, a la vez que se encapsula en un marco analítico culturalista, desconectado en exceso de las realidades sociales concretas a las que esos discursos se supone que aluden.

En este sentido, se está en pleno acuerdo con Daniel Hiernaux (2006; 2018) cuando, tomando como referencia el diagnóstico que Carlos Reynoso (2000) hace de los estu- 
dios culturales, advierte de la escasa efectividad de estos en su pretensión de confluir con aproximaciones interesadas en aportar conocimiento sólido sobre el turismo.

Igualmente, se comparte en líneas generales con Hiernaux (2018) su apreciación acerca de la necesidad de distinguir entre el proceso (el turismo) y su aplicación. Aunque en este caso se apuntan dudas: por lo que toca a los impactos ambientales causados por la industria turística internacional, ¿una variación significativa de su modus operandi, acaso no implicaría una completa redefinición no ya de prácticas concretas, sino del propio hecho turístico? No se quiere incurrir en un tipo de sinécdoque por la cual se enjuicie el todo a partir de una fracción, pero si de verdad se tomara en serio la censura y erradicación de las dinámicas perniciosas ocasionadas por esa parte: ¿qué quedaría entonces del todo?

\section{Conclusión}

Cuando la definición del contexto se convierte en un asunto más político que científico, pues de su conquista depende qué obtendrá cada uno de los actores implicados, las ciencias sociales se arriesgan a prostituir sus principios en entornos en los que la opinión no se estudia, se fabrica. Penetradas por influencers y emisores de fake news, las sociedades modernas enfrentan la investigación del turismo ante retos de envergadura:

a) La posibilidad de salvaguardar espacios de independencia desde los cuales llevar a cabo análisis rigurosos e innovadores, es decir, capaces de cuestionar el orden presentado como inevitable. Se requiere asumir que la apuesta unilateral por las lógicas del trabajo acumulativo, comentadas más arriba, no se adaptan bien a la naturaleza de la investigación científico-social, y esto afecta a la calidad y originalidad de los estudios turísticos.

b) Revisar, criticar y adoptar, cuando sea conveniente, nuevas perspectivas y metodologías que contribuyan a enriquecer la comprensión del turismo, como hecho social total, aglutinador de procesos económicos, políticos, culturales y ecológicos de alcance global. Por eso, se sugiere promover una formación transdisciplinar que integre (y supere) las tradicionales oposiciones micromacro, cualitativo-cuanti-tativo, elementos simbólicos-estructuras materiales, naturaleza-cultura, estabilidad-cambio, por mencionar algunas. 
c) Defender las convicciones éticas del investigador, pero no instrumentalizarlas como prescripciones morales o ideológicas del trabajo científico-social. Es decir, se recomienda vincular el principal compromiso ético de la investigación con su función desenmascaradora del entramado de relaciones de poder, mentiras, intereses privados, convenciones o costumbres justificadoras del statu quo, que emanan del actual contexto sociotécnico y que, como si de una nube tóxica se tratara, lo encapotan.

Como en el relato bíblico, hay que confiar en que la verdad traerá la libertad, aunque no necesariamente la justicia social, o al menos no de manera inmediata. Dicho de otro modo, los resultados obtenidos con la investigación del turismo pueden ayudar a pensar con más claridad y autonomía la dinámica del entramado turístico ( $y$, dada su actual relevancia, del conjunto del sistema social), pero no habilitan para prescribir cambios y alternativas irrefutables de este sistema.

La constatación empírica del sufrimiento objetivo provocado por determinados comportamientos (turismo sexual, explotación laboral en el sector, entre otros), así como la difusión de esos hallazgos, tienen una poderosa capacidad transgresora, agitando conciencias e, indirectamente, modificando la opinión pública sobre problemas antes invisibilizados. De esa manera, quienes se benefician de esos comportamientos tendrán más problemas a la hora de obtener los inputs de legitimidad social requeridos para perpetuar sus conductas.

La aspiración de una sociedad más justa pertenece al ámbito de la lucha y la reivindicación política, la cual sería deseable que se sostuviera en argumentaciones racionales, ancladas en conocimiento procedente de prácticas investigadoras honestas y rigurosas. Se propone, en definitiva, aceptar que el discernimiento de lo que está bien o mal, de lo que es estimable o repudiable, hermoso o feo, no deriva de los hallazgos obtenidos a través del trabajo científico, sino de la contraposición de juicios morales que, en el mejor de los casos, mantienen un diálogo constructivo, tomando como referencia tales descubrimientos.

Lo anterior no debe soslayar la responsabilidad del investigador cuando define y acota la realidad que conforma su objeto de estudio, establece un tipo de relación cognoscitiva con esa realidad y planifica el acceso a la misma, pues de los compromisos adquiridos dependerá la clase de luz que proyecten sus hallazgos y las partes de la realidad que se iluminen (en un segundo momento, esas decisiones tendrán repercusiones éticas y políticas). 
El argumento desarrollado no considera que la política y la investigación del turismo sean esferas independientes, todo lo contrario, el poder impregna la mayoría de las interacciones sociales. Lo que afirma es que la acción política, al margen de que se exprese por medio de estrategias subversivas más o menos beligerantes, se encauce por canales institucionalizados y transparentes, o se gestione desde grupúsculos restringidos y opacos, debe disociarse de la praxis científico-social.

El investigador, por supuesto, debe comunicar sus hallazgos, asesorar y formular recomendaciones, en coherencia con los resultados obtenidos en sus estudios. Así, enriquecerá la calidad de los debates que han de mantener los actores implicados en el espacio influido por el turismo, pero en esa tarea no debe olvidar las limitaciones con las que se topa el discurso científico; si lo hiciese, tendría enseguida que renunciar a la autonomía y la credibilidad conferida por el hecho de ser productor de un tipo de explicaciones que, por definición, pretenden reducir en la medida de lo posible el ruido ideológico y la parcialidad política.

Orientado por sus convicciones, también puede optar por implicarse en los procesos de transformación social, pero entonces su rol de investigador se diluiría tras el de activista o gestor, y esos sí son dos ámbitos que han de separarse.

La instrumentalización (manipulación) política y propagandística que las élites del poder han hecho (y siguen haciendo) del trabajo científico, y del científico-social con especial énfasis, no debe combatirse con una contra-instrumentalización dirigida por sectores organizados de la sociedad civil. La primera víctima de esa disputa sería la legitimidad de las ciencias sociales y de los estudios turísticos.

Otra cuestión es aceptar que esa pérdida resulta asumible, si a cambio se incrementan las opciones de expansión de la justicia social. En ese caso, el disimulo (el hacer pasar una cosa por otra) puede entenderse como parte de una estrategia política más amplia. Cada uno puede hacer su personal valoración al respecto. Se concluye entonces con algunas aclaraciones finales y limitaciones relativas a todo lo expuesto.

Las alusiones realizadas a corrientes positivistas y posmarxistas son susceptibles de ser sometidas a infinidad de matices. La distancia que separa a unas y otras no forma un continuo $y$, en consecuencia, no se quiere dar a entender que los desarrollos teórico-metodológicos que agrupan estos enfoques agoten las muy variadas propuestas epistemológicas de las ciencias sociales y de los estudios turísticos, simplemente la atención que se les ha prestado ha sido útil para construir un hilo 
conductor. De hecho, la apuesta por la transdisciplinariedad surge de una confianza (asumida a priori) en la capacidad de las posiciones holísticas y eclécticas para confluir con miradas en las que se reconozca un espíritu crítico (y esa asunción, se admite, puede identificarse como un sesgo de la exposición).

El carácter ensayístico de este texto no está dirigido por una voluntad pontificadora, desde luego no es esa la intención del autor; al contrario, trata de alimentar una discusión ya existente, de gran interés, en la que tienen cabida diversas sensibilidades acerca de la naturaleza de la investigación en turismo. Se invita a los lectores a participar en este debate abierto, planteando razonamientos y soluciones diferentes.

\section{Referencias}

Aitchison, C.C. (2005). Feminist and gender perspectives in tourism studies: the social-cultural nexus of critical and cultural theories. Tourist Studies, 5(3), 207-224. https://doi.org/10.1177/1468797605070330

Ateljevic, I., Pritchard, A. y Morgan, N. (Eds.). (2007a). The critical turn in tourism studies: innovative research methodologies. Elsevier.

Ateljevic, I., Pritchard, A. y Morgan, N. (Eds.). (2007b). Editors' introduction: promoting an academy of hope in tourism enquiry. En I. Ateljevic, A. Pritchard y N. Morgan (Eds.), The critical turn in tourism studies: innovative research methodologies (pp. 1-8). Elsevier.

Beard, L., Scarles, C. y Tribe, J. (2016). Mess and method: using ANT in tourism research. Annals of Tourism Research, 60, 97-110. https://doi.org/10.1016/ j.annals.2016.06.005

Becken, S. y Hay, J. (2007). Tourism and climate change. Risks and opportunities. Channel View Publications.

Castillo-Nechar, M. y Panosso, A. (2010). Epistemología del turismo. Estudios críticos. Editorial Trillas.

Cole, S. (Ed.). (2018). Gender equality and tourism. Beyond empowerment. University of the West of England.

Coles, T., Hall, C. M. y Duval, D. T. (2006). Tourism and post-disciplinary enquiry. Current Issues in Tourism, 9(4-5), 293-319. https://doi.org/10.2167/cit327.0 
Darbellay, F. y Stock, M. (2012). Tourism as complex interdisciplinary research object. Annals of Tourism Research, 39(1), 441-458. https://doi.org/10.1016/j.annals.2011.07.002

De Kadt, E. (1979). Tourism: passport to development? Perspectives on the social and cultural effects of tourism in developing countries. Oxford University Press.

Estivill, J. (1979). Lloret de Mar: destruccions i resistencies d'un poble en mans del turisme. Papers, Revista de Sociologia, 10, 175-201. https://doi.org/10.5565/ rev/papers/v10n0.1107

Fennell, D. (2006). Tourism ethics. Channel View Publications.

Figueroa-Domecq, C., Pritchard, A., Segovia-Pérez, M., Morgan, N. y Villacé-Molinero, T. (2015). Tourism gender research: a critical accounting. Annals of Tourism Research, 52, 87-103. https://doi.org/10.1016/j.annals.2015.02.001

Galán, J.J., Martín, A., Ruiz, J. y Mandly, A. (1977). Costa del Sol. Retrato de unos colonizados. Cuadernos de Campo Abierto Ediciones.

Gaviria, M. (1974). España a go-go: turismo charter y neocolonialismo del espacio. Turner.

Greenwood, D. J. (1989). Culture by the pound: an anthropological perspective on tourism as cultural commoditization. En V. Smith (Ed.), Hosts and guests: the anthropology of tourism (pp. 171-186). University of Pennsylvania Press. https://doi.org/10.9783/9780812208016

Habermas, J. (1981). Theorie des kommunikativen Handelns. Suhrkamp.

Hiernaux, D. (2006). Geografía del turismo. En D. Hiernaux y A. Lindón (Coords.), Tratado de geografía humana (pp. 397-428). Anthropos y Universidad Autónoma Metropolitana-Iztapalapa. https://bit.ly/3cUH9|4

Hiernaux, D. (2018). Más de tres décadas de investigación turística en México: algunas reflexiones. Dimensiones Turísticas, 2(2), 123-132. https://doi.org/10.47557/ RIEX6572

Hollinshead, K. (1992). 'White' gaze, 'red' people. Shadow visions: the disidentification of 'Indians' in cultural tourism. Leisure Studies, 11(1), 43-64. https://doi. org/10.1080/02614369100390301 
Hollinshead, K. (1999). Surveillance of the worlds of tourism: Foucault and the eyeof-power. Tourism Management, 20(1), 7-23. https://doi.org/10.1016/S02615177(98)00090-9

Honneth, A. (1987). Critical theory. En A. Giddens y J. Turner (Eds.), Social theory today (pp. 347-382). Stanford University Press.

Horkheimer, M. (1947). Eclipse of reason. Oxford University Press.

Horkheimer, M. y Adorno, Th. (1947). Dialektik der Aufklärung. Querido.

Huete, R. (2019). La digitalización de la economía y el fin del turismo. En A. Álvarez-Sousa, A. Mantecón e I. Puertas-Cañaveral (Eds.), Sociología del turismo (pp. 299-321). Centro de Investigaciones Sociológicas. https://bit.ly/3naoA0R

Igoe, J., Neves, K. y Brockington, D. (2010). A spectacular eco-tour around the historic bloc: theorizing the convergence of biodiversity conservation and capitalist expansion. Antipode, 42(3), 486-512. https://doi.org/10.1111/j.14678330.2010.00761.x

Jurdao, F. (1979). España en venta. Compra de suelos por extranjeros y colonización de campesinos en la Costa del Sol. Ayuso.

Kuhn, T.S. (1962). The structure of scientific revolutions. University of Chicago Press.

Kuhn, T.S. (1977). The essential tension. Selected studies in scientific tradition and change. University of Chicago Press.

Landsberger, H. A. (1958). Hawthorne revisited: management and the worker, its critics, and developments in human relations in industry. Cornell University.

Leiper, N. (2000). An emerging discipline. Annals of Tourism Research, 27(3), 805-809. https://doi.org/10.1016/S0160-7383(99)00118-8

Mantecón, A. (2016). La opinión pública sobre el turismo. Crítica y propuesta para una nueva orientación. Estudios y Perspectivas en Turismo, 25(2), 110-123. https://bit.ly/3cmaG76

Mantecón, A. y Velasco, M. (2020). Beyond tourismphobia: conceptualizing a new framework to analyze attitudes towards tourism. En C. Ribeiro-de-Almeida, A. Quintano, M. Simancas, R. Huete y Z. Breda (Eds.), Handbook of research on the impacts, challenges, and policy responses to overtourism (pp. 60-74). IGI Global. https://bit.ly/3ld0Jfp

Marx, K. (1888). Thesen über Feuerbach. J. H. W. Dietz. 
Marx, K. y Engels, F. (1848). Manifest der Kommunistischen Partei. Bildungs-Gesellschaft für Arbeiter.

Merton, R. K. (1949). Social theory and social structure: toward the codification of theory and research. Free Press.

Milano, C. (2017). Otherness anthropologies: toward ibero-american anthropologies of tourism. American Anthropologist, 119(4), 736-741. https://doi. org/10.1111/aman.12957

Mills, C. W. (1959). The sociological imagination. Oxford University Press.

Muecke, S. y Wergin, C. (2014). Materialities of tourism in the twenty-first century: a very brief introduction. Tourist Studies, 14(3), 227-230. https://doi. org/10.1177/1468797614536307

Nash, D. (1977). Tourism as a form of imperialism. En V. Smith (Ed.), Hosts and guests: the anthropology of tourism (pp. 37-52). University of Pennsylvania Press. https://doi.org/10.9783/9780812208016.37

Osorio, M. y Korstanje, M. E. (2017). Reflexiones en torno a la situación de la investigación turística. Dimensiones Turísticas, 1(1), 13-30. https://doi.org/10.47557/ JJLL9743

Picard, D. y Robinson, M. (2012). Emotion in motion: tourism, affect and transformation. Routledge.

Pritchard, A., Morgan, N. y Ateljevic, I. (2011). Hopeful tourism: a new transformative perspective. Annals of Tourism Research, 38(3), 941-963. https://doi. org/10.1016/j.annals.2011.01.004

Reynoso, C. (2000). Apogeo y decadencia de los estudios culturales. una visión antropológica. Gedisa. https://bit.ly/3n9U33k

Rosa, H. (2010). Alienation and acceleration: towards a critical theory of late-modern temporality. Nova Southeastern University Press.

Scott, D. (Coord.). (2008). Climate change and tourism. Responding to global challenges. The United Nations Environment Program \& World Meteorological Organization. https://bit.ly/33oZ4wW

Sheller, M. y Urry, J. (2006). The new mobilities paradigm. Environment and Planning A: Economy and Space, 38(2), 207-226. https://doi.org/10.1068/a37268 
Tobar-Bustamante, L. E., Castillo-Nechar, M., Mendoza-Valdés, R. y Tamayo-Salcedo, A. L. (2015). La visión crítica en el construir del conocimiento turístico. Revista de Análisis Turístico, 19, 33-44. https://bit.ly/3kCCKpr

Tribe, J. (1997). The indiscipline of tourism. Annals of Tourism Research, 24(3), 638657. https://doi.org/10.1016/S0160-7383(97)00020-0

Tribe, J. (2000). Indisciplined and unsubstantiated. Annals of Tourism Research, 27(3), 809-813. https://doi.org/10.1016/S0160-7383(99)00122-X

Tribe, J. (2007). Critical tourism: rules and resistance. En I. Ateljevic, A. Pritchard y N. Morgan (Eds.), The critical turn in tourism studies (pp. 29-39). Elsevier. https://doi.org/10.1016/B978-0-08-045098-8.50007-6

Tribe, J. (2009). Philosophical issues in tourism. Channel View. https://doi.org/ $10.21832 / 9781845410988$

Turner, L. y Ash, J. (1975). The golden hordes: international tourism and the pleasure periphery. Constable.

Urry, J. (2007). Mobilities. Polity Press.

Wang, Y., Yu, Q. y Fesenmaier D. R. (2002). Defining the virtual tourist community: implications for tourism marketing. Tourism Management, 23(4), 407-417. https://doi.org/10.1016/S0261-5177(01)00093-0

Wang, D., Park, S. y Fesenmaier, D. R. (2012). The role of smartphones in mediating the touristic experience. Journal of Travel Research, 51(4), 371-387. https://doi.org/ $10.1177 / 0047287511426341$

Wilson, E., Small, J. y Harris, C. (2012). Editorial introduction: beyond the margins? The relevance of critical tourism and hospitality studies. Journal of Hospitality and Tourism Management, 19(1), 48-51. https://doi.org/10.1017/jht.2012.2

Znaniecki, F. (1927). The object matter of sociology. American Journal of Sociology, 32(4), 529-584. https://doi.org/10.1086/214184 